\title{
Dynamique de structuration spatio-temporelle des populations de familles de macroinvertébrés dans un continuum lac de barrage-effluent-fleuve issu de périmètre irrigué. Bassin de la Volta (Burkina Faso)
}

\author{
SANOGO Souleymane* et KABRE Tinkoudgou Jean André* \\ *Laboratoire de Recherche et de Formation en Pêche et Faune (LaRFPF/ IDR), Université de Bobo-Dioulasso, BP. 1091 \\ Bobo 01, Burkina Faso ; email of main contact : ankab226@yahoo.fr
}

Original submitted in on 20 th February 2014. Published online at www.m.elewa.org on 30 th June 2014. http://dx.doi.org/10.4314/jab.v78i1.9

\section{RÉSUMÉ}

Objectif : Un échantillonnage mensuel de macroinvertébrés benthiques a été effectué au barrage hydroagricole de Boura dans le bassin de la Volta (Burkina Faso) pendant la période de février à juillet 2012 en vue de décrire la structuration des taxons inféodés aux habitats tributaires de ce système d'irrigation.

Méthodologie et résultats : La collecte des échantillons s'effectué sur 6 stations placés sur le littoral (station I), le sublittoral (Station II), l'effluent (stations III, IV qui sont situées au niveau du périmètre irrigué et la station V loin de ce périmètre) et le fleuve Mouhoun (station $\mathrm{VI}$ ); la méthode de collecte s'inspire de la méthode conventionnelle de la "Directive Cadre sur l'Eau de l'Union Européenne (DCE)» préconisée pour l'échantillonnage des macroinvertébrés benthiques. L'étude révèle une communauté composée majoritairement d'insectes (plus de 75\%) et diversement repartit. D'une part au niveau du transect littoralsublittoral dans le lac de barrage, 23 familles de macroinvertébrés ont pu être échantillonnés; toutes se trouvaient au niveau du littoral et seulement 10 au niveau du sublittoral. Ces deux zones du lac de barrage comportent la même faune malacologique à savoir des individus de la famille des Unionidés, des Lymnéidés, des Ampullariidés, des Planorbidés, des Valvatidés et des Bulinidés. L'étude révèle par ailleurs que la différence entre les indices de diversité biologique de Shannon de ces deux zones s'accentue pendant le mois de juillet, période de pluie au niveau du bassin. D'autre part au niveau du continuum lac de barrage-effluentfleuve, 35 familles ont été identifiées dont 27 dans le lac de barrage et des stations proches du périmètre irrigué et 32 au niveau de la station $V$ de l'effluent loin du périmètre irrigué et la station VI du fleuve. La présence d'individus appartenant à la famille des Baetidés et des Ephémérellidés (ordre des Ephéméroptères) au niveau du fleuve permet de conclure une possible récupération du cours d'eau car cet ordre d'insectes est considéré comme sensible aux perturbations des cours d'eau.

Conclusion et application de résultats : il est conclu de cette étude que les aménagements hydro-agricoles ont une influence sur la structuration spatio-temporelle des macroinvertébrés. Les résultats de cette étude peuvent par ailleurs être utilisés pour conserver la biodiversité et la restauration de ces écosystèmes continuellement impactés.

Mots clé : macroinvertébrés, structuration, continuum, barrage hydroagricole, effluent, fleuve Mouhoun, Bassin de la Volta 


\section{ABSTRACT}

Objective: A monthly sampling of benthic macroinvertebrates was done at the hydro-agricultural of dam Boura in the basin of the Volta (Burkina) during the period of February to July 2012 in order to describe the structuring of insect succession along with changes occurring in habitats of this irrigation system.

Methodology and results: The samples of insects were collected from 6 stations placed on the shoreline (Station I), the adjoining zone to the coastline (Station II), the sewage channal (stations III, IV that is located at midsection of the irrigated area and the Station $\mathrm{V}$ far away from this area) and the Mouhoun stream (Station VI); the sampling method used conventional method the European Union named " Directive Cadre sur l'Eau (DCE) " advised for the sampling of the benthics macroinvertebrates. The survey revealed a community composed in majority of insects (more of $75 \%$ ) and sparsely distributed. On one hand at the shoreline and the coastal- adjoining zone in the lake of dam, 23 families of macroinvertebrates were sampled; they mostly belong to the shoreline except for 10 families identified in the adjoining zone to the coastline. These two zones of the dam shelter the same malacological fauna consisting of the families of Unionidae, Lymneidas, Ampullariidae, Planorbidae, Valvatidae and Bulinidae. The survey revealed otherwise that the difference between the biological diversity indices of Shannon of these two zones was more pronounced during the month of July, period of rain in the basin., concerning the dam-sewage channal-river continuum, a total of 35 families were sampled: 27 from dam water and stations near the irrigated zones; and 32 been collected from the station of the sewage channel far from the irrigated perimeter and from the stream. The identification of individuals belonging to the family of the Baetidae and the Ephemerellidae (order of the Ephemeroptera) in the river water highlights a possible reconstruction of biodiversity in the river because both two families are indicator fertile water.

Conclusion and application of results: it is concluded of this survey that the agricultural hydro amenities have an influence on the spatio-temporal structuring of the macroinvertebrates. The results of this survey can be used otherwise to keep biodiversity and the restoration of these ecosystems continually impacted

Key words: macroinvertebrates, succession, continuum, hydro-agricultural dams, irrigation, river, Mouhoun, Volta basin.

\section{INTRODUCTION}

En Afrique subsaharienne, la construction des retenues d'eau à but souvent hydroagricole sur les lits des cours d'eau a engendré une fragmentation et des modifications écologiques des milieux naturels ; c'est le cas du Burkina Faso où près de 1700 retenues d'eau ont été construites (Cecchi et al., 2009). Ces modifications des écosystèmes sont des réalités inévitables, ceux qui emmènent Vénot et Cecchi (2011) à les considérer comme des « socioécosystèmes ». Leight et al. (2010) et Sass et al. (2010) indiquent que l'intensification agricole est une des causes principales de dégradation des écosystèmes aquatiques. L'évaluation de l'impacte des différentes pressions anthropiques sur les ressources naturelles est aujourd'hui centrée sur l'analyse des modifications de structuration des organismes constituant la biodiversité ; c'est ainsi que la polluo-sensibilité différentielle des macroinvertébrés définit par Marzin et al. (2012) reste un outil de choix dans l'évaluation de la qualité biologique de ces écosystèmes. Les travaux antérieurs sur l'évaluation des organismes, les macroinvertébrés benthiques notamment, au Burkina Faso regroupent ceux de Déjoux (1977) sur le lac Bam, Guenda (1996) au niveau du fleuve Mouhoun, Kabré et al. (2002) lac de barrage de la Comoé. Cependant l'inventaire des taxons de macroinvertébrés inféodés aux habitats tributaires des aménagements agricoles ainsi que leurs structurations n'ont pas été élucidés par ces études antérieures. Cette étude menée au niveau d'un barrage hydro-agricole de Boura dans le bassin de la Volta se propose de faire l'inventaire des macroinvertébrés benthiques et de démontrer leur distribution sur le continuum lac de barrage-effluentFLEUVE. 


\section{MÉTHODOLOGIE}

Milieu d'étude: Le bassin de la volta est le plus important du Burkina Faso ; il s'étend au centre et à l'ouest du pays sur une superficie de $178000 \mathrm{~km}^{2}$. II est constitué par trois sous-bassins dont fait parti le sousbassin du Mouhoun avec comme principal cours d'eau le fleuve Mouhoun. Notre zone d'étude est le lac de barrage hydroagricole de Boura $\left(11^{\circ} 02^{\prime} \mathrm{N}, 2^{\circ} 30^{\prime} \mathrm{W}\right)$ construit sur un affluent du fleuve Mouhoun (figure 1). Ce lac de barrage est construit pour subvenir aux besoins des activités des populations de la commune rurale de 22 villages et d'environ 40000 habitants. En effet, historiquement la digue du barrage avait été mise en place en 1950 par les villageois et fut reconstruite en 1983 par l'Office National des Barrages et de l'Irrigation (ONBI). Elle a une capacité de 4,2 millions de $\mathrm{m}^{3}$. Ce réservoir permet d'alimenter un périmètre irrigué (riz et maïs) de 62 ha ; la retenue permet aussi d'entretenir une culture de rente de contre saison d'environ 30 hectares (cultures maraîchères et de maïs) situés le long de ses rives en amont du déversoir. Les eaux excédentaires de la retenue d'eau se déversent en aval dans l'affluent qui les draine vers le fleuve et de passage collecte les eaux usées de la plaine irriguée; c'est ainsi que nous l'appelons effluent. Les plantes aquatiques majoritairement présentes au niveau de la retenue et son l'effluent est l'Azzola, le Ceratophyllum, les Nénuphars, et des graminées du genre Oriza.

Collecte des macroinvertébrés : L'échantillonnage mensuel s'est déroulé de février 2012 à juillet 2012 au niveau du barrage et de l'effluent issus des périmètres irrigués en aval de la digue. Les stations d'investigation se répartissent comme suit (figure 1):

- $\quad$ Station I : littoral du lac du barrage (moins d'un mètre de la rive) ;

- $\quad$ Station II : sublittoral du lac de barrage (plus d'un mètre de la rive)

- $\quad$ Station III : début de l'effluent à $50 \mathrm{~m}$ en aval du déversoir du barrage ;
- $\quad$ Station IV : sur l'effluent en plein périmètre irrigué, à $2 \mathrm{~km}$ en aval du déversoir ;

Station V : sur l'effluent à $14 \mathrm{~km}$ en aval du déversoir (zone intermédiaire entre le périmètre irrigué et le fleuve Mouhoun);

- $\quad$ Station VI : au niveau du fleuve Mouhoun à 36 $\mathrm{km}$ de la digue du lac de barrage.

La période de collecte des macroinvertébrés aquatiques au niveau des différentes stations est marquée par une intensification de l'irrigation en saison sèche de novembre à mai. Les périmètres de culture en aval du lac de barrage sont irrigués par des vannes qui drainent l'eau par un système de canalisation. Vue l'importance de la couverture végétale au niveau des zones d'échantillonnage, les 12 coups de filets préconisés par la Directive Cadre Européenne de l'Eau (AFNOR, 2009) en tenant compte des potentiels habitats ont été répartis également entre la vase et les zones d'herbiers. Les espèces de surfaces ont aussi été capturées. Au niveau de chaque station, les macroinvertébrés ont été collectés à l'aide d'un filet de type Surber d'ouverture $25 \mathrm{~cm}$ et d'un filet troubleau d'une ouverture de $30 \mathrm{~cm}$ de diamètre. Le filet Surber qui a servi à collecter les espèces benthiques est tiré sur une longueur de $1 \mathrm{~m}$. La surface de balayage correspond donc à $3 \mathrm{~m}^{2}$ par échantillonnage. Après capture, les espèces de macroinvertébrés sont triées et conservées dans de l'alcool $90^{\circ}$. Le reste du benthos contenant des macroinvertébrés non visibles à l'œil nus, est conservé dans des bocaux avec de l'alcool $90^{\circ}$ pour un tri au laboratoire. Le filet troubleau a servi à collecter les espèces inféodées aux chevelures racinaires des macrophytes : pendant 30 secondes, le filet est passé sous les plantes. Le contenu du filet composé de parties de plantes, de macroinvertébrés et de boue, est rincés à l'eau, puis les macroinvertébrés sont retirés et conservés à l'alcool $90^{\circ}$, le reste étant conservé dans des bocaux avec de l'alcool $90^{\circ}$ pour un tri au laboratoire. Ce filet trouble eau a également servi à la capture des espèces de surface. 

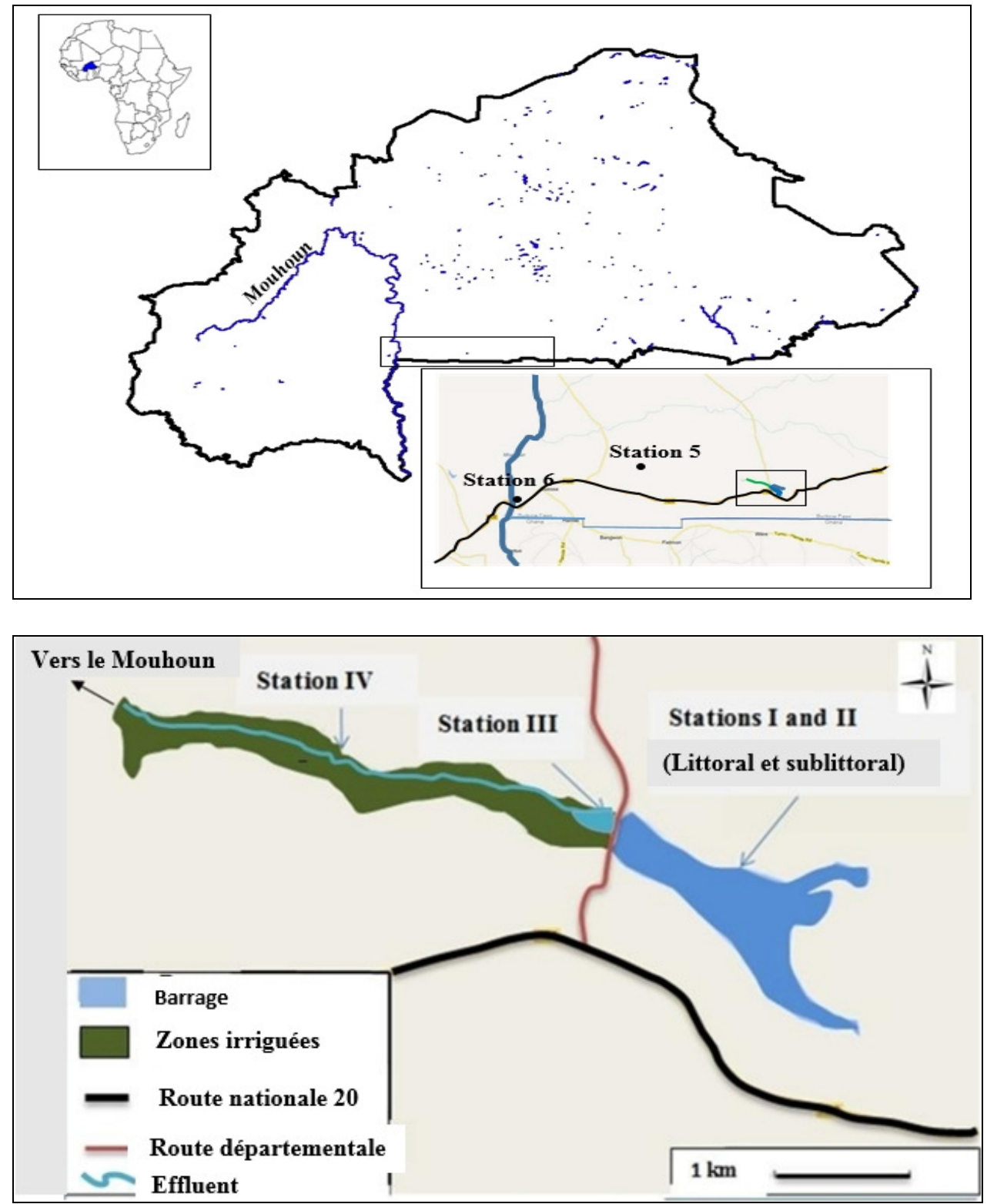

Figure 1 : Localisation des différentes stations d'échantillonnage.

Identification des macroinvertébrés : Les insectes ont été identifiés grâce aux clés d'identification de Levêque et Durand (1981), Merrit et Cummins (1984), Tachet et al. (2000) et de Moisan (2006). Les mollusques ont été identifiés à l'aide de la clé de Moisan (2010) et de la publication de Brown (1980). Les annélides ont été identifiés avec les clés fournis avec Introduction Pratique à la Systématique des Organismes des Eaux Continentales Françaises : 3 . Annélides Oligochètes de Lafon (1983). Le niveau taxonomique atteint dans notre étude est la famille. En effet ce niveau taxonomique permet une discrimination globale des sites dans des études de bioindication (AFNOR, 2004 ; AFNOR, 2009 ; Moisan et Pelletier, 2008).

Analyses statistiques : L'indice de diversité biologique de Shannon (Shannon et Weaver, 1949) est donné par la formule

$$
H^{\prime}=-\sum_{i=1}^{S} p_{i} \ln p_{i}
$$

$\mathrm{p}_{\mathrm{i}}=\mathrm{Ni} / \mathrm{N}$

$\mathrm{Ni}$ : nombre d'individus d'un taxon donné, i allant de 1 à S (nombre total de taxons).

$\mathrm{N}$ : nombre total d'individus. 
H' est minimal $(=0)$ si tous les individus du peuplement appartiennent à un seul et même taxon, H' est également minimal si, dans un peuplement chaque espèce est représentée par un seul individu, excepté un taxon qui est représentée par tous les autres individus du peuplement. L'indice est maximal quand tous les individus sont répartis d'une façon équitable sur tous les taxons. Généralement cet indice est calculé en considérant l'espèce comme niveau taxonomique. Moisan et Pelletier (2008), estime que la structuration de communautés de macroinvertébrés benthiques des cours, regroupant les variables métriques et des indices tel l'indice de diversité de Shannon peuvent être définis au niveau taxonomique qu'est la famille. Ce niveau taxonomique a en effet permis à Zouggaghe et Moali (2009) de montrer que des zones polluées ont des indices faibles par rapport aux zones non polluées dans un cours d'eau algérien. En référence à la démarche de ces auteurs nous avons opté pour la famille comme niveau taxonomique pour le calcul de l'indice de diversité.

\section{RÉSULTATS}

Discrimination des populations de macroinvertébrés des stations d'échantillonnage : Ces échantillonnages ont permis de récolter 27177 individus dont 8644 individus au niveau du littoral du barrage et 1272 au niveau du sublittoral respectivement station I et station II (Tableau 1). En aval de la digue du barrage dans l'effluent 5618 individus ont été récoltés au niveau de la station III, 5572 au niveau de la station IV et 3051 à la station V; au niveau du fleuve Mouhoun 3020 individus à la station VI (Tableau 2). Ces macroinvertébrés se composent d'Insectes, de Crustacés, de Vers et de Mollusques regroupés en 35 familles, parmi lesquelles une famille d'Amphipodes et une de Cladocères ; toutes des crustacées non identifiés à cause leur petite taille et leur fragilité à la conservation. Les stations I, II, III et IV considérées comme théoriquement impactés par les activités agricoles comptent 27 familles et au niveau des stations $V$ et $V I$ qui sont loin des périmètres irrigués, 32
Le logiciel Past (PAleontological STatistics, Version 2.16, Hammer et al., 2001) a été utilisé pour construire des dendrogrammes de classification hiérarchisée. La distance euclidienne est utilisée comme distance d'assemblage. Les indices de diversité de Shannon ont été calculé avec le logiciel Past. L'utilisation de la plus petite différence significative de Fisher avec $a=0,05$ (PPDSF) a permis de comparer les indices de diversité entre les différents au niveau d'une même station d'une part et entre les stations d'autre part. La différence est significative lorsque PPDSF est inférieur à la valeur absolue de la différence entre deux moyennes.

La comparaison faunistique des stations a été réalisée en calculant le coefficient de similitude (Cs) de Sørensen (1948) : $\mathrm{Cs}=\frac{2 \mathrm{C}}{\mathrm{a}+\mathrm{b}} \div 100$

Cs : coefficient de similitude de Sorensen

a : nombre de taxon dans le milieu $A$

$b$ : nombre de taxon dans le milieu $B$

$c$ : nombre de taxon commun à $A$ et $B$

familles. Les insectes sont les plus dominants avec $89,23 \%$ au niveau du littoral et $65,64 \%$ pour le sublittoral ; $85,81 \%, 86,49 \%$ et $75,22 \%$ respectivement aux stations III, IV et $V$ dans l'effluent ; finalement $89,11 \%$ au niveau du fleuve, station VI.

La classification hiérarchisée des stations basée sur la similarité des familles de macroinvertébrés (Figure 2) permet de regrouper ces stations en quatre :

- $\quad$ Groupe 1 : la station du littoral du lac de barrage (station I)

- Groupe 2 : les stations au niveau des périmètres irrigués (stations II et III)

- Groupe 3 : les stations après les périmètres irrigués (stations IV et $\mathrm{V}$ )

- Groupe 4 : la station du sublittoral du lac de barrage (station $\mathrm{VI}$ ) 
Sanogo et Kabre. J. Appl. Biosci. 2014. Dynamique de structuration spatio-temporelle des populations de familles de macroinvertébrés dans un continuum lac de barrage -effluent-fleuve, Volta Burkina Faso

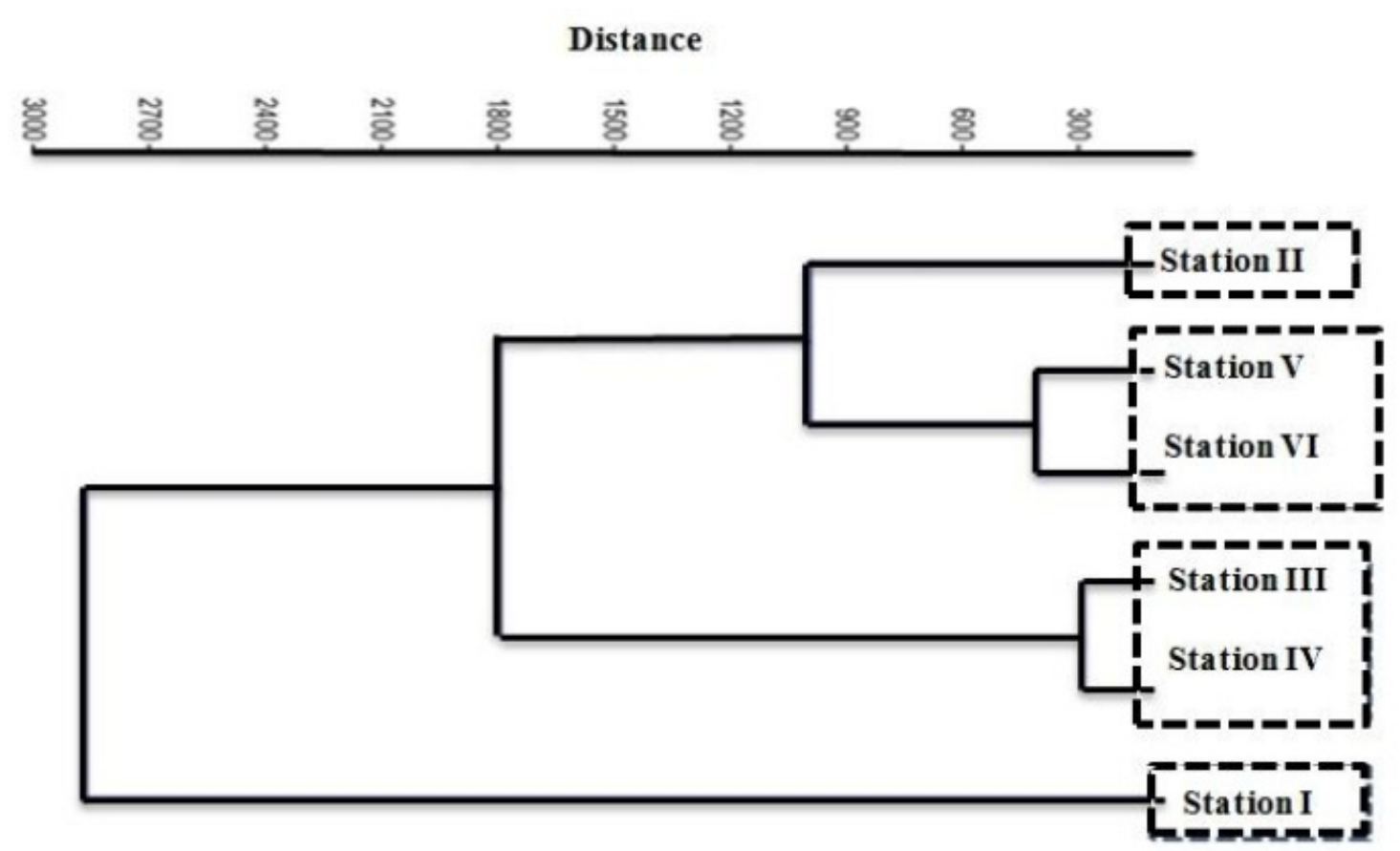

Figure 2 : Classification hiérarchiques des différentes stations basée sur la similarité des assemblages de familles de macroinvertébrés. 
Sanogo et Kabre. J. Appl. Biosci. 2014. Dynamique de structuration spatio-temporelle des populations de familles de macroinvertébrés dans un continuum lac de barrage -effluent-fleuve, Volta Burkina Faso

Tableau 1 : Nombre d'individus par famille de macroinvertébrés échantillonnés au niveau de deux stations d'observation du barrage de Boura pendant la période de février à juillet 2012.

\begin{tabular}{|c|c|c|c|c|c|c|c|c|c|c|c|c|}
\hline \multirow[t]{2}{*}{ Macroinvertébrés } & \multicolumn{6}{|c|}{ Station I (littoral lac de Barrage) } & \multicolumn{6}{|c|}{ Station II (sublittoral lac de barrage) } \\
\hline & Fév. & Mar & Avr & Mai & Jui & Jul & Fév. & Mar & Avr. & Mai & Jui & Jul \\
\hline \multicolumn{13}{|l|}{ Coléoptères } \\
\hline Hydrophilidae & 986 & 418 & 845 & 704 & 356 & 0 & 0 & 17 & 47 & 88 & 8 & 0 \\
\hline Dytiscidae & 358 & 313 & 263 & 188 & 208 & 59 & 0 & 20 & 0 & 16 & 21 & 0 \\
\hline Gyrinidae & 0 & 0 & 0 & 0 & 0 & 0 & 0 & 0 & 0 & 0 & 0 & 0 \\
\hline Carabidae & 3 & 11 & 0 & 2 & 0 & 0 & 0 & 0 & 0 & 0 & 0 & 0 \\
\hline Noteridae & 419 & 687 & 398 & 244 & 104 & 69 & 85 & 90 & 157 & 73 & 46 & 0 \\
\hline Chrysomelidae & 0 & 0 & 0 & 0 & 0 & 0 & 0 & 0 & 0 & 0 & 0 & 0 \\
\hline Elmidae & 0 & 0 & 0 & 0 & 0 & 0 & 0 & 0 & 0 & 0 & 0 & 0 \\
\hline \multicolumn{13}{|l|}{ Hétéroptères } \\
\hline Notonectidae & 16 & 25 & 12 & 18 & 22 & 15 & 0 & 0 & 0 & 0 & 0 & 0 \\
\hline Naucoridae & 7 & 23 & 0 & 6 & 0 & 9 & 0 & 0 & 0 & 0 & 0 & 0 \\
\hline Belostomatidae & 23 & 27 & 20 & 6 & 9 & 13 & 0 & 8 & 11 & 15 & 7 & 5 \\
\hline Nepidae & 38 & 17 & 29 & 15 & 31 & 6 & 0 & 6 & 3 & 1 & 3 & 1 \\
\hline Gerridae & 0 & 23 & 0 & 0 & 13 & 19 & 0 & 0 & 0 & 0 & 0 & 0 \\
\hline Herbridae & 22 & 0 & 6 & 18 & 21 & 0 & 0 & 0 & 0 & 0 & 0 & 0 \\
\hline Hydrometridae & 12 & 6 & 22 & 3 & 9 & 8 & 0 & 0 & 0 & 0 & 0 & 0 \\
\hline Veliidae & 0 & 0 & 0 & 0 & 0 & 0 & 0 & 0 & 0 & 0 & 0 & 0 \\
\hline \multicolumn{13}{|l|}{ Ephéméroptères } \\
\hline Baetidae & 0 & 0 & 0 & 0 & 0 & 0 & 0 & 0 & 0 & 0 & 0 & 0 \\
\hline Ephemerellidae & 0 & 0 & 0 & 0 & 0 & 0 & 0 & 0 & 0 & 0 & 0 & 0 \\
\hline \multicolumn{13}{|l|}{ Odonates } \\
\hline Gomphidae & 15 & 3 & 17 & 11 & 4 & 10 & 0 & 0 & 0 & 0 & 0 & 0 \\
\hline Libellulidae & 13 & 8 & 22 & 10 & 19 & 6 & 0 & 0 & 0 & 0 & 0 & 0 \\
\hline Coenagrionidae & 10 & 1 & 6 & 2 & 7 & 6 & 0 & 0 & 0 & 0 & 0 & 0 \\
\hline \multicolumn{13}{|l|}{ Diptères } \\
\hline Chironomidae & 46 & 112 & 98 & 31 & 26 & 6 & 12 & 49 & 22 & 17 & 7 & 0 \\
\hline Syrphidae & 2 & 5 & 13 & 0 & 0 & 0 & 0 & 0 & 0 & 0 & 0 & 0 \\
\hline \multicolumn{13}{|l|}{ Crustacés } \\
\hline Potamidae & 0 & 0 & 0 & 0 & 0 & 0 & 0 & 0 & 0 & 0 & 0 & 0 \\
\hline Amphipodes & 0 & 0 & 0 & 0 & 0 & 0 & 0 & 0 & 0 & 0 & 0 & 0 \\
\hline $\begin{array}{l}\text { Cladocères } \\
\text { Vers }\end{array}$ & 0 & 0 & 0 & 0 & 0 & 0 & 0 & 0 & 0 & 0 & 0 & 0 \\
\hline
\end{tabular}


Sanogo et Kabre. J. Appl. Biosci. 2014. Dynamique de structuration spatio-temporelle des populations de familles de macroinvertébrés dans un continuum lac de barrage -effluent-fleuve, Volta Burkina Faso

\begin{tabular}{|c|c|c|c|c|c|c|c|c|c|c|c|c|}
\hline Hirudinae & 3 & 14 & 3 & 4 & 20 & 11 & 4 & 0 & 0 & 0 & 6 & 9 \\
\hline Lumbricidae & 0 & 0 & 0 & 0 & 0 & 0 & 0 & 0 & 0 & 0 & 0 & 0 \\
\hline Naididae & 0 & 0 & 0 & 0 & 0 & 0 & 0 & 0 & 0 & 0 & 0 & 0 \\
\hline \multicolumn{13}{|l|}{ Mollusques } \\
\hline Bulinidae & 114 & 56 & 51 & 29 & 14 & 5 & 29 & 73 & 83 & 18 & 0 & 1 \\
\hline Unionidae & 4 & 7 & 15 & 3 & 12 & 6 & 0 & 3 & 0 & 10 & 5 & 0 \\
\hline Pleuroceridae & 0 & 0 & 0 & 0 & 0 & 0 & 0 & 0 & 0 & 0 & 0 & 0 \\
\hline Lymneidae & 140 & 84 & 20 & 20 & 3 & 8 & 44 & 52 & 12 & 3 & 0 & 8 \\
\hline Ampullariidae & 10 & 58 & 44 & 8 & 2 & 8 & 27 & 6 & 6 & 0 & 0 & 0 \\
\hline Planorbidae & 21 & 41 & 14 & 2 & 11 & 26 & 0 & 22 & 6 & 3 & 0 & 0 \\
\hline Valvatidae & 15 & 6 & 11 & 5 & 0 & 3 & 3 & 4 & 0 & 0 & 0 & 0 \\
\hline
\end{tabular}

Tableau 2 : Nombre d'individus par famille de Macroinvertébrés échantillonnés au niveau des stations situées sur l'effluent issu du lac de barrage de Boura de février juillet 2012.

\begin{tabular}{|c|c|c|c|c|c|c|c|c|c|c|c|c|c|c|c|c|c|c|c|c|c|c|c|c|}
\hline \multirow[b]{3}{*}{ Macroinvertébrés } & \multicolumn{12}{|c|}{ Périmètres irrigués } & \multirow{2}{*}{\multicolumn{6}{|c|}{$\begin{array}{c}\text { Zone intermédiaire } \\
\text { Station V }\end{array}$}} & \multirow{2}{*}{\multicolumn{6}{|c|}{$\begin{array}{c}\text { Fleuve Mouhoun } \\
\text { Station VI }\end{array}$}} \\
\hline & \multicolumn{6}{|c|}{ Station III } & \multicolumn{6}{|c|}{ Station IV } & & & & & & & & & & & & \\
\hline & Fev & Mar & Avr & Mai & Jui & Jul & Fev & Mar & Avr & Mai & Jui & Jul & Fev & Mar & Avr & Mai & Jui & Jul & Fev & Mar & Avr & Mai & Jui & Jul \\
\hline \multicolumn{25}{|l|}{ Coléoptères } \\
\hline Hydrophilidae & 512 & 311 & 355 & 356 & 511 & 17 & 47 & 469 & 188 & 608 & 600 & 0 & 0 & 165 & 182 & 149 & 0 & 13 & 299 & 0 & 234 & 54 & 13 & 7 \\
\hline Dytiscidae & 274 & 279 & 183 & 120 & 109 & 28 & 320 & 250 & 148 & 221 & 108 & 0 & 0 & 183 & 193 & 224 & 85 & 15 & 232 & 230 & 159 & 127 & 42 & 0 \\
\hline Gyrinidae & 0 & 0 & 0 & 0 & 0 & 0 & 0 & 0 & 8 & 0 & 0 & 13 & 0 & 0 & 4 & 0 & 17 & 0 & 9 & 0 & 7 & 0 & 3 & 0 \\
\hline Carabidae & 21 & 0 & 0 & 0 & 3 & 0 & 0 & 0 & 16 & 3 & 0 & 0 & 0 & 3 & 0 & 11 & 0 & 0 & 0 & 0 & 0 & 0 & 0 & 0 \\
\hline Noteridae & 131 & 232 & 109 & 121 & 44 & 18 & 166 & 314 & 130 & 104 & 49 & 18 & 0 & 35 & 25 & 22 & 25 & 0 & 3 & 0 & 23 & 0 & 3 & 0 \\
\hline Chrysomelidae & 13 & 6 & 41 & 12 & 12 & 0 & 0 & 14 & 25 & 0 & 0 & 0 & 12 & 25 & 7 & 17 & 4 & 0 & 26 & 3 & 15 & 18 & 0 & 0 \\
\hline $\begin{array}{l}\text { Elmidae } \\
\text { Hétéroptères }\end{array}$ & 0 & 14 & 2 & 3 & 13 & 0 & 11 & 15 & 12 & 0 & 13 & 24 & 27 & 3 & 9 & 0 & 0 & 3 & 44 & 12 & 15 & 11 & 0 & 18 \\
\hline Notonectidae & 0 & 0 & 0 & 0 & 0 & 0 & 0 & 0 & 0 & 0 & 0 & 0 & 0 & 0 & 33 & 10 & 7 & 0 & 0 & 0 & 0 & 0 & 0 & 0 \\
\hline Naucoridae & 3 & 0 & 0 & 8 & 0 & 0 & 2 & 4 & 0 & 10 & 5 & 26 & 0 & 0 & 0 & 0 & 0 & 0 & 0 & 0 & 0 & 0 & 0 & 0 \\
\hline Belostomatidae & 18 & 31 & 11 & 41 & 23 & 25 & 18 & 28 & 13 & 12 & 20 & 34 & 0 & 15 & 21 & 19 & 46 & 21 & 9 & 23 & 19 & 11 & 23 & 41 \\
\hline Nepidae & 21 & 39 & 10 & 22 & 43 & 3 & 29 & 28 & 36 & 9 & 7 & 10 & 2 & 73 & 32 & 54 & 19 & 6 & 6 & 7 & 15 & 0 & 0 & 0 \\
\hline Gerridae & 14 & 0 & 23 & 13 & 11 & 7 & 31 & 20 & 6 & 14 & 39 & 22 & 0 & 39 & 7 & 12 & 41 & 37 & 36 & 49 & 61 & 40 & 21 & 32 \\
\hline Herbridae & 37 & 12 & 41 & 10 & 0 & 0 & 18 & 24 & 2 & 11 & 0 & 0 & 0 & 9 & 26 & 13 & 0 & 0 & 4 & 7 & 2 & 18 & 0 & 0 \\
\hline Hydrometridae & 9 & 0 & 3 & 9 & 2 & 0 & 9 & 2 & 6 & 11 & 0 & 1 & 0 & 7 & 12 & 3 & 0 & 0 & 9 & 1 & 6 & 2 & 0 & 0 \\
\hline
\end{tabular}




\begin{tabular}{|c|c|c|c|c|c|c|c|c|c|c|c|c|c|c|c|c|c|c|c|c|c|c|c|c|}
\hline $\begin{array}{l}\text { Veliidae } \\
\text { Ephéméroptères }\end{array}$ & 44 & 7 & 17 & 0 & 11 & 0 & 33 & 7 & 0 & 12 & 0 & 0 & 2 & 0 & 11 & 0 & 5 & 10 & 12 & 1 & 14 & 11 & 41 & 32 \\
\hline Baetidae & 0 & 0 & 0 & 0 & 0 & 0 & 0 & 0 & 0 & 0 & 0 & 0 & 0 & 0 & 0 & 0 & 0 & 0 & 1 & 0 & 3 & 0 & 2 & 0 \\
\hline $\begin{array}{l}\text { Ephemerellidae } \\
\text { Odonates }\end{array}$ & 0 & 0 & 0 & 0 & 0 & 0 & 0 & 0 & 0 & 0 & 0 & 0 & 0 & 0 & 0 & 0 & 0 & 0 & 2 & 0 & 1 & 0 & 0 & 4 \\
\hline Gomphidae & 9 & 6 & 18 & 17 & 1 & 4 & 3 & 5 & 8 & 10 & 9 & 17 & 0 & 15 & 3 & 2 & 14 & 0 & 22 & 19 & 3 & 6 & 7 & 18 \\
\hline Libellulidae & 16 & 22 & 6 & 13 & 6 & 23 & 14 & 9 & 22 & 13 & 5 & 11 & 0 & 21 & 17 & 9 & 8 & 4 & 36 & 38 & 16 & 25 & 17 & 23 \\
\hline $\begin{array}{l}\text { Coenagrionidae } \\
\text { Diptères }\end{array}$ & 2 & 8 & 13 & 9 & 7 & 10 & 9 & 11 & 3 & 5 & 2 & 1 & 0 & 6 & 11 & 5 & 4 & 2 & 14 & 21 & 24 & 10 & 3 & 11 \\
\hline Chironomidae & 41 & 18 & 77 & 10 & 36 & 0 & 58 & 20 & 16 & 44 & 9 & 0 & 0 & 11 & 36 & 15 & 26 & 12 & 65 & 19 & 82 & 21 & 11 & 7 \\
\hline $\begin{array}{l}\text { Syrphidae } \\
\text { Crustacés }\end{array}$ & 6 & 11 & 8 & 6 & 0 & 0 & 23 & 11 & 11 & 17 & 0 & 0 & 0 & 8 & 3 & 13 & 0 & 0 & 0 & 0 & 0 & 0 & 0 & 0 \\
\hline Potamidae & 0 & 0 & 0 & 0 & 0 & 0 & 0 & 0 & 0 & 0 & 0 & 0 & 0 & 0 & 9 & 3 & 0 & 5 & 0 & 0 & 0 & 0 & 0 & 0 \\
\hline Amphipodes & 0 & 0 & 0 & 0 & 0 & 0 & 0 & 0 & 0 & 0 & 0 & 0 & 0 & 0 & 0 & 0 & 0 & 0 & 0 & 0 & 14 & 0 & 0 & 0 \\
\hline $\begin{array}{l}\text { Cladocères } \\
\text { Vers }\end{array}$ & 0 & 0 & 0 & 0 & 0 & 0 & 0 & 0 & 0 & 0 & 0 & 0 & 0 & 0 & 0 & 0 & 0 & 0 & 7 & 0 & 5 & 0 & 0 & 0 \\
\hline Hirudinae & 8 & 0 & 11 & 9 & 16 & 31 & 16 & 18 & 7 & 12 & 31 & 21 & 0 & 0 & 7 & 3 & 21 & 38 & 0 & 0 & 1 & 6 & 0 & 0 \\
\hline Lumbricidae & 0 & 5 & 14 & 4 & 4 & 0 & 41 & 18 & 22 & 31 & 15 & 8 & 0 & 0 & 0 & 0 & 0 & 0 & 0 & 0 & 0 & 0 & 0 & 0 \\
\hline $\begin{array}{l}\text { Naididae } \\
\text { Mollusques }\end{array}$ & 0 & 0 & 0 & 0 & 0 & 0 & 0 & 0 & 0 & 0 & 0 & 0 & 0 & 17 & 26 & 32 & 11 & 0 & 0 & 0 & 0 & 0 & 0 & 0 \\
\hline Bulinidae & 55 & 18 & 5 & 52 & 0 & 0 & 16 & 0 & 91 & 26 & 12 & 0 & 0 & 0 & 0 & 0 & 0 & 0 & 0 & 0 & 0 & 0 & 0 & 0 \\
\hline Unionidae & 40 & 33 & 28 & 30 & 18 & 13 & 23 & 39 & 15 & 13 & 25 & 11 & 35 & 18 & 39 & 15 & 29 & 10 & 54 & 32 & 54 & 27 & 22 & 16 \\
\hline Pleuroceridae & 0 & 0 & 0 & 0 & 0 & 0 & 0 & 0 & 0 & 0 & 0 & 0 & 63 & 21 & 2 & 107 & 21 & 0 & 0 & 0 & 0 & 0 & 0 & 0 \\
\hline Lymneidae & 55 & 34 & 25 & 22 & 12 & 22 & 32 & 14 & 15 & 13 & 0 & 0 & 0 & 25 & 20 & 13 & 0 & 0 & 15 & 5 & 0 & 0 & 0 & 0 \\
\hline Ampullariidae & 11 & 18 & 7 & 2 & 0 & 23 & 10 & 9 & 15 & 11 & 0 & 0 & 0 & 7 & 16 & 11 & 0 & 0 & 6 & 2 & 3 & 0 & 0 & 0 \\
\hline Planorbidae & 27 & 11 & 15 & 6 & 0 & 11 & 0 & 14 & 6 & 0 & 3 & 0 & 0 & 21 & 39 & 24 & 0 & 0 & 3 & 13 & 11 & 9 & 0 & 0 \\
\hline Valvatidae & 23 & 14 & 20 & 19 & 10 & 16 & 34 & 23 & 13 & 8 & 14 & 8 & 5 & 11 & 12 & 8 & 6 & 6 & 9 & 8 & 7 & 0 & 0 & 0 \\
\hline
\end{tabular}


Variabilité structurelle des peuplements de macroinvertébrés

A l'échelle du lac de barrage : Au niveau du sublittoral, 13 familles à savoir les Hydrophilidés, les Dytiscidés, les Noteridés, les Bélostomatidés, les Népidés, les Chironomidés tous des Insectes, les Vers comprenant la familles des Hirudinés, et les Mollusques composés des familles de Bulinidés, de Unionidé, de Lymnéidés, de Ampullariidés, de Planorbidés et de Valvatidés, échantillonnées se retrouvent aussi au niveau du littoral qui compte 10 familles en plus à savoir les Carabidés, les Notonectidés, les Naucoridés, les Gérridés, les Herbridés, les Hydrométridés, les Gomphidés, les Libéllulidés, les Coenogrionidés et les Syrphidés tous des Insectes. Le constat est que toutes les familles de
Mollusques colonisent aussi bien le littoral que le sublittoral.

En outre, l'indice de similarité de Sørensen est de $72,22 \%$ ce qui indique une différence entre ces deux stations du point de vue de leur diversité. En effet ces différences sont mises en évidence par le test de Fisher (PPDF) avec $\alpha=5 \%$ (Tableau 3). L'utilisant de cette valeur (PPDF 0,05 ) permet de constater une différence entre le mois de juillet et les autres mois au niveau du littoral et une différence entre le mois de mars et les autres mois au niveau du sublittoral. En comparant les droites de régressions linéaires des indices par mois des deux stations (Figure 3) pendant la période qui s'étend de février à juillet, on note une croissance de l'indice au niveau du littoral pendant qu'il décroit au niveau sublittoral.

Tableau 3 : Comparaison de la variation mensuelle de l'indice de diversité de Shannon suivant un transect allant du littoral au sublittoral du lac de barrage de Boura ; la Plus Petite Différence Significative de Fisher (PPDS avec $\alpha=5 \%$ ) est utilisée.

\begin{tabular}{lcc}
\hline & $\begin{array}{c}\text { Littoral } \\
\text { (Station I) }\end{array}$ & $\begin{array}{c}\text { Sublittoral } \\
\text { (Station II) }\end{array}$ \\
\hline \hline Février & 1,804 & 1,546 \\
Mars & 1,988 & 2,037 \\
Avril & 1,792 & 1,554 \\
Mai & 1,577 & 1,718 \\
Juin & 1,951 & 1,664 \\
Juillet & 2,501 & 1,326 \\
\hline \hline Moyenne & 1,935 & 1,640 \\
\hline \hline PPDSF0.05 & 0,289 & \\
\hline \hline
\end{tabular}

A l'échelle du continuum lac de barrage-effluentfleuve : L'indice de Sørensen une similarité minimum de $80 \%$ entre ces quatre stations (tableau 4) : le lac de barrage, les périmètres irrigués, la zone intermédiaire et le fleuve. L'utilisation de la plus petite différence significative de Fisher (tableau 5) met en évidence d'une part des différences entre les indices de diversité de Shannon mensuels au niveau de chaque station et d'autre part des différences des indices mensuels entre les stations pour un mois choisi. En outre une comparaison entre les valeurs moyennes des trois zones écologiques barrage, effluent et fleuve indique une différence significative entre les barrages et les deux autres pendant la période de février, avril et mai (tableau 5 bis). Au niveau du barrage (station I et II) et de l'effluent (station III et IV, zones théoriquement impactées) les indices de diversité de Shannon du mois de juillet diffèrent de ceux des autres mois.

Des 35 familles de macroinvertébrés présents dans nos échantillons, 18 sont communes aux 4 zones à savoir les Hydrophilidés, les Dytiscidés, les Notéridés, de l'ordre des Coléoptères, les Bélostomatidés, les Népidés, les Gerridés, les Herbridés, les Hydrométridés de l'Ordre des Hétéroptères, les Gomphidés, les Libéllulidés et les Coenagrionidés de l'ordre des Odonates, les Chironomidés de l'ordre des Diptères, les Hirudinés de l'embranchement des Vers les 5 familles de Mollusques à savoir les Unionidés, les Lymnéidés, les Ampullariidés, les Planorbidés et les Valvatidés. 


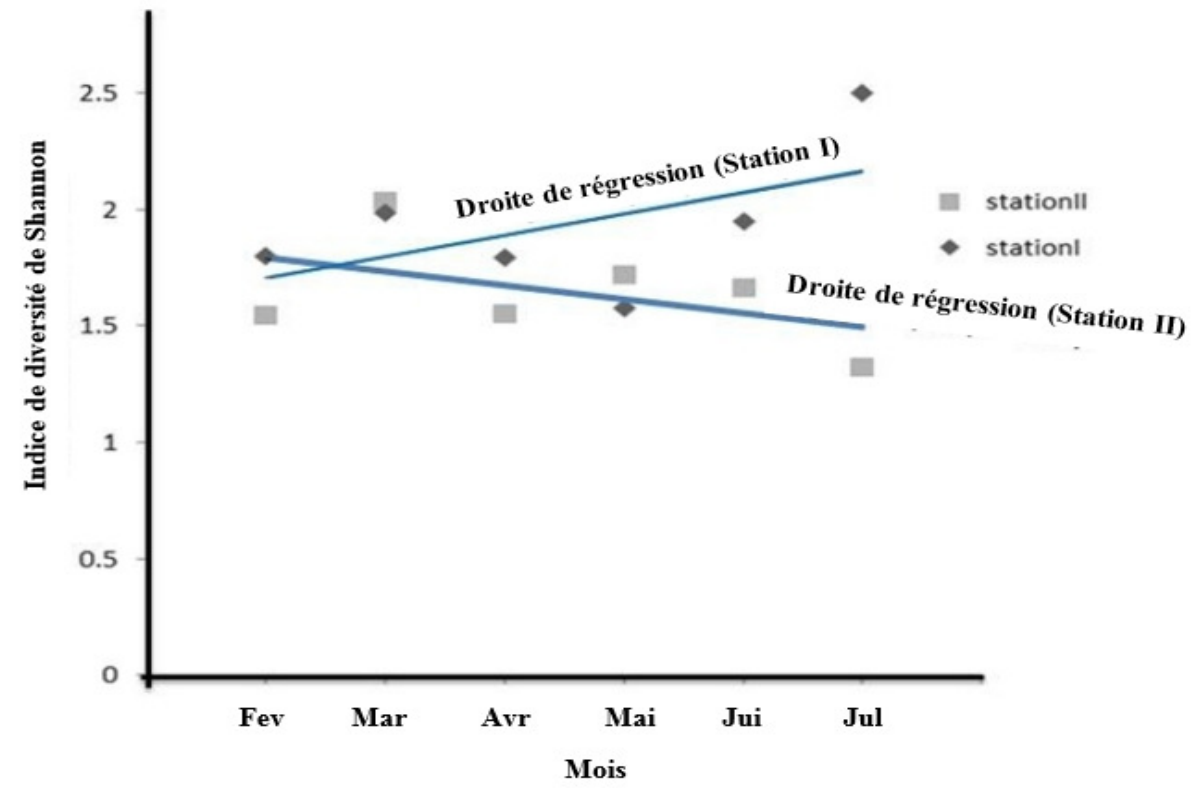

Figure 3 : Comparaison des régressions linéaires des indices de diversité de Shannon obtenu au niveau du littoral et du sublittoral dans le barrage de Boura de février à juillet 2012.

Tableau 4 : Indice de similarité de Sørensen entre les différents sites du lac de barrage au fleuve

\begin{tabular}{lcccc}
\hline \hline & $\begin{array}{c}\text { Lac de barrage } \\
\text { (stations I et II) }\end{array}$ & $\begin{array}{c}\text { Périmètres irrigués } \\
\text { (stations III et IV) }\end{array}$ & $\begin{array}{c}\text { Zone intermédiaire } \\
\text { (station V) }\end{array}$ & Fleuve (station VI) \\
\hline \hline $\begin{array}{l}\text { Lac de barrage } \\
\text { (station I et II) }\end{array}$ & $100 \%$ & $92 \%$ & $82,35 \%$ & $85,71 \%$ \\
$\begin{array}{l}\text { Périmètres irrigués } \\
\text { (stations III et IV) }\end{array}$ & $92 \%$ & $100 \%$ & $98,18 \%$ & $81,48 \%$ \\
$\begin{array}{l}\text { Zone intermédiaire } \\
\text { (station V) }\end{array}$ & $82,35 \%$ & $98,18 \%$ & $100 \%$ & $86,79 \%$ \\
Fleuve (station VI) & $85,71 \%$ & $81,48 \%$ & $86,79 \%$ & $100 \%$ \\
\hline \hline
\end{tabular}

Tableau 5 : Comparaison de la variation mensuelle de l'indice de diversité de Shannon le long du continuum lac de barrage-effluent-fleuve Mouhoun ; la comparaison utilise la Plus Petite Différence Significative de Fisher (PPDSF avec $\mathrm{a}=0,05$ ).

\begin{tabular}{lcccc}
\hline \hline & $\begin{array}{c}\text { Lac de barrage } \\
\text { (station I et II) }\end{array}$ & $\begin{array}{c}\text { Périmètres irrigués } \\
\text { (station III et IV) }\end{array}$ & $\begin{array}{c}\text { Station intermédiaire } \\
\text { (V) }\end{array}$ & $\begin{array}{c}\text { Fleuve (station } \\
\text { VI) }\end{array}$ \\
\hline \hline Février & 1,9 & 2,438 & 1,456 & 2,204 \\
Mars & 2,092 & 2,117 & 2,479 & 1,994 \\
Avril & 1,876 & 2,484 & 2,602 & 2,347 \\
Mai & 1,648 & 2,072 & 2,426 & 2,293 \\
Juin & 1,989 & 1,679 & 2,559 & 2,231 \\
Juillet & 2,551 & 2,830 & 2,317 & 2,14 \\
\hline \hline Moyenne & 2,009 & 2,270 & 2,306 & 2,201 \\
\hline \hline PPDSF 0.05 & 0,340 & 0,340 & 0,340 & 0,340 \\
\hline \hline
\end{tabular}


Sanogo et Kabre. J. Appl. Biosci. 2014. Dynamique de structuration spatio-temporelle des populations de familles de macroinvertébrés dans un continuum lac de barrage -effluent-fleuve, Volta Burkina Faso

Tableau 5 bis : Synthèse des différences entre les valeurs mensuelles des indices de diversité de Shannon entre les stations (station I à VI ; tableau 5) d'observation le long du continuum lac de barrage effluent - fleuve Mouhoun. (en gras : écarts dont les valeurs absolues sont supérieures à PPDSF 0.05 ).

\begin{tabular}{|c|c|c|c|c|c|}
\hline & & Barrage & Périmètres irrigué & Zone intermédiaire & Fleuve \\
\hline \multirow{4}{*}{ Juillet } & Barrage & 0 & & & \\
\hline & Périmètres irrigués & 0,279 & 0 & & \\
\hline & Zone intermédiaire & $-0,234$ & $-0,513$ & 0 & \\
\hline & Fleuve & $-0,411$ & $-0,69$ & $-0,177$ & 0 \\
\hline \multirow{4}{*}{ Juin } & Barrage & 0 & & & \\
\hline & Périmètres irrigués & $-0,31$ & 0 & & \\
\hline & Zone intermédiaire & 0,57 & 0,88 & 0 & \\
\hline & Fleuve & 0,242 & 0,552 & $-0,328$ & 0 \\
\hline \multirow{4}{*}{ Mai } & Barrage & 0 & & & \\
\hline & Périmètres irrigués & 0,424 & 0 & & \\
\hline & Zone intermédiaire & 0,778 & 0,354 & 0 & \\
\hline & Fleuve & 0,645 & 0,221 & $-0,133$ & 0 \\
\hline \multirow{4}{*}{ Avril } & Barrage & 0 & & & \\
\hline & Périmètres irrigués & 0,608 & 0 & & \\
\hline & Zone intermédiaire & 0,726 & 0,118 & 0 & \\
\hline & Fleuve & 0,471 & $-0,137$ & $-0,255$ & 0 \\
\hline \multirow{4}{*}{ Mars } & Barrage & 0 & & & \\
\hline & Périmètres irrigués & 0,025 & 0 & & \\
\hline & Zone intermédiaire & 0,387 & 0,362 & 0 & \\
\hline & Fleuve & $-0,098$ & $-0,123$ & $-0,485$ & 0 \\
\hline \multirow{4}{*}{ Février } & Barrage & 0 & & & \\
\hline & Périmètres irrigués & 0,538 & 0 & & \\
\hline & Zone intermédiaire & $-0,444$ & $-0,982$ & 0 & \\
\hline & Fleuve & 0,304 & $-0,234$ & 0,748 & 0 \\
\hline
\end{tabular}

Nous avons choisi de faire de la Figure 4 une représentation spatiale des familles (au nombre de 17) qui ne sont pas communes aux différentes stations; ceci permet de mieux magnifier le contraste ou la discrimination entre les zones écologiques. Sur le transect allant du lac de barrage (stations I et II) aux deux première stations de l'effluent (stations III et IV) on constate une présence permanente de Naucoridés et de Bulinidés; ces stations, proches des activités agricoles comptent au total 27 familles de macroinvertébrés. Deux familles de Coléoptères, présents au niveau des stations de l'effluent jusqu'au fleuve sont absent au niveau du lac de barrage: il s'agit de la famille des
Chrysomélidés et de celle des Elmidés. Au niveau des stations III et IV, on a une présence endémique des Lumbricidés et des Véliidés. On rencontre également la famille des Véliidés au niveau du fleuve. Les Potamidés, les Naididés et les Pleuroceridés se retrouvent seulement au niveau de la station intermédiaire (zone dont les berges ne sont pas utilisées). La zone intermédiaire et le fleuve considérés comme des stations non impactées comptent 32 familles. Finalement Les Gyrinidés commencent à apparaitre au niveau de la dernière station de l'effluent (station VI) jusqu'au fleuve Mouhoun (station VI). Ce fleuve (station VI) se caractérise par la présence des Baetidés, des 
Ephémérellidés, des Amphipodes et des Cladocères, tous absents des autres stations. Ces autres stations se distinguent du fleuve par la présence des Carabidés et des Syrphidés.

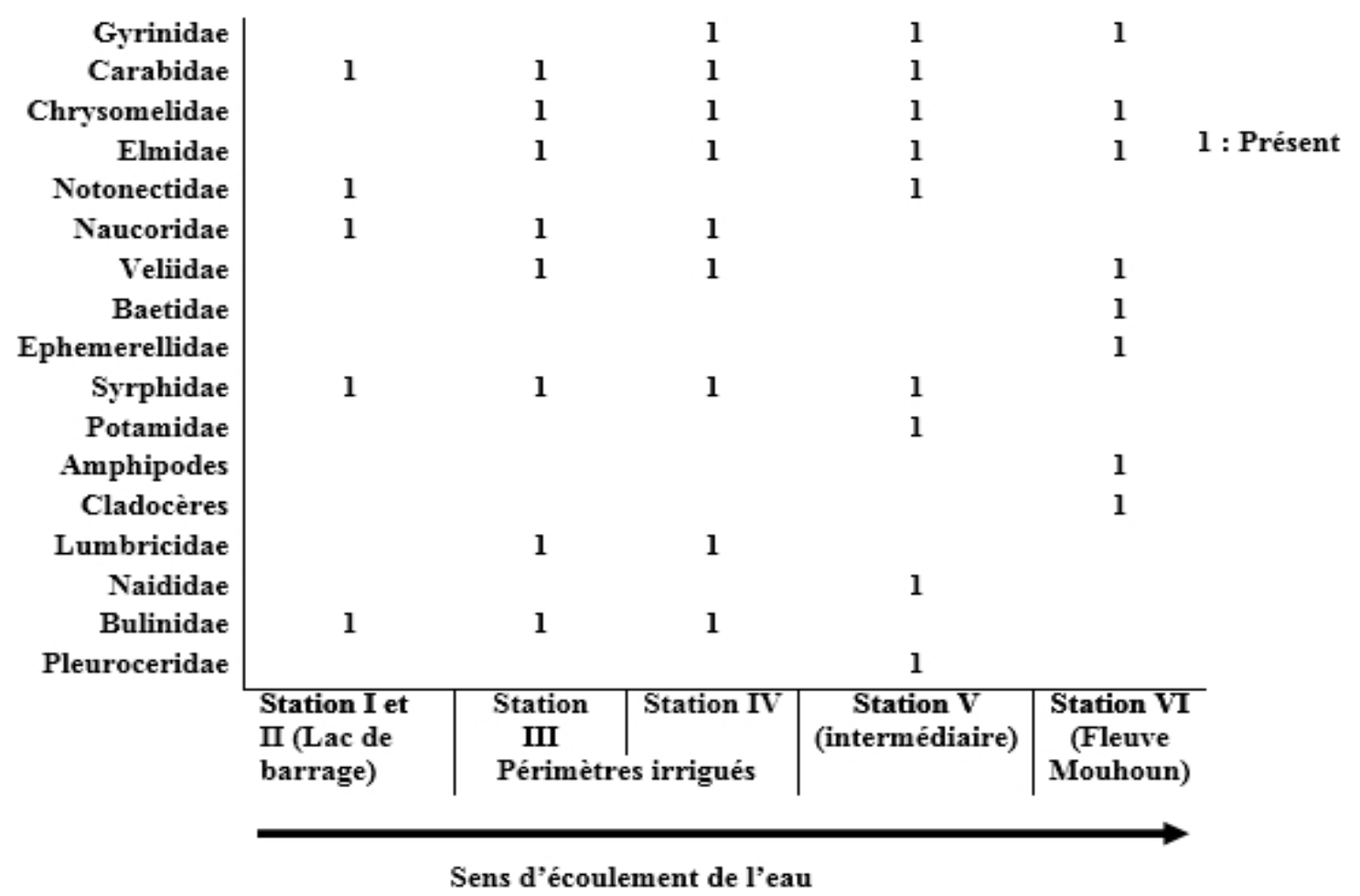

Figure 4 : Répartition spatiale de macroinvertébrés non communes aux stations situés sur l'effluent du lac de barrage jusqu'au fleuve Mouhoun

\section{DISCUSSION}

Les écosystèmes aquatiques du Burkina subissent depuis longtemps des pressions anthropiques à travers l'utilisation de divers produits dont la majorité provient des activités agricoles (GIRE, 2001). Au niveau des zones d'irrigation, Davis et al., (2011) ont indiqué les effets néfastes des pesticides sur les communautés de macroinvertébrés. Au niveau du barrage du lac de Boura des stations de l'effluent au niveau du périmètre irrigué, 27 familles de macroinvertébrés ont été échantillonnées. Ce résultat est en adéquation avec des résultats des travaux de Diomandé et al, (2009) et ceux de Foto et al., (2011) qui ont obtenu chacun 28 familles respectivement en Côte d'Ivoire et au Cameroun au niveau des zones impactées par les activités agricoles et les rejets urbains. Par contre, au niveau des zones non impactées (zone intermédiaire et fleuve) 32 familles ont été échantillonnées. Ces résultats sont en deçà des 59 familles obtenues par Foto et al., (2010) dans la caractérisation d'un site de référence au niveau d'un cours d'eau. La distinction observée entre les stations du lac de barrage par la figure 2, confirmée par la comparaison des droites de régression de la figure 3 indique une forte colonisation des zones du littoral par les macroinvertébrés par rapport au sublittoral. Pourtant le sublittoral est présenté comme une zone abritant les taxons sensibles dans des systèmes eubiotiques (Mazzela et al., 2009) ; d'où une zone où la diversité naturelle des invertébrés en général est la plus forte (Wiederholm, 1980). Des études de Kouamé et al., (2011) indiquaient une concentration des macroinvertébrés d'un barrage au niveau des plantes aquatiques. Au niveau du lac de barrage de Boura, l'utilisation des berges pour les activités agricoles a engendré une concentration de plantes aquatiques dans le littoral d'où cette concentration de macroinvertébrés à ce niveau. Dans ce transect littoral-sublittoral, les droites de régression (Figure 3) des indices de diversité mensuels de Shannon, indiquent que la fin de la saison 

familles de macroinvertébrés dans un continuum lac de barrage -effluent-fleuve, Volta Burkina Faso

sèche équivaut à une grande diversité des familles de macroinvertébrés au niveau du littoral pendant qu'elle diminue au niveau du sublittoral. Le calcul de la plus petite différence de Fisher ressort une différence entre les indices de diversité des deux stations au mois de juillet, qui correspond à la pleine saison pluvieuse au niveau du bassin de la Volta. Malgré cette dissemblance, on constate que le littoral et le sublittoral comportent les mêmes populations de mollusques. En effet, dans l'étude de la distribution bathymétrique des mollusques, Mouthon (1993) avait constaté que les mollusques avaient la capacité d'occuper ces deux zones au niveau d'un lac. Pequeux (2006) les trouvait aussi dans les zones profondes aquatiques surtout les fonds marins. Parmi ceux échantillonnés au niveau du lac de barrage de Boura, on constate une prolifération de Bulinidés; ce constat avait été fait par Poda et al. (2006) au niveau des aménagements hydrauliques couramment appelés petits barrages au Burkina. Au niveau du continuum lac de barrage-effluent-fleuve, 18 des 35 familles de macroinvertébrés sont communes à toutes les stations. La figure 2 regroupe sur ce continuum, les stations du périmètre irrigué de l'effluent et celle de la station intermédiaire à celle du fleuve. Ces résultats indiquent que les modifications de l'habitat déterminent la structure des communautés de macroinvertébrés. En effet, aucune station n'est parfaitement identique à une autre sur ce continuum. Des différences significatives sont observées lorsque les écarts entre les indices mensuels sont comparés à la différence significative de Fisher, surtout au niveau des écarts entre l'indice au niveau du lac de barrage et les autres stations (tableau 5bis) pendant les mois de février, avril et mai. En effet, au niveau de cet

\section{CONCLUSION}

Les basses eaux sont des périodes d'intense activités agricoles au niveau des barrages hydroagricoles du bassin de la Volta. Les communautés de macroinvertébrés subissent par conséquent les agressions de cette intensification agricoles. Au niveau du lac de barrage de Boura et du continuum établit avec le fleuve Mouhoun, l'étude de la structuration de cette faune sentinelle révèle une communauté composée majoritairement d'insectes (plus de 75\%) et diversement repartit. Au niveau du transect littoral-sublittoral dans le lac de barrage, 23 familles de macroinvertébrés ont pu être échantillonnés; tous se trouvaient au niveau du littoral et seulement 10 au niveau du sublittoral. L'étude habitat (lac de barrage), on constate que la moyenne arithmétique des indices de diversité mensuels est le plus basse dans ce continuum pendant ces mois. En outre, la différence observée entre les zones de cultures et les zones non impactées pendant le mois de juillet (mois de fortes pluies) indique une augmentation notable de la diversité au niveau de ces zones (cours d'eau) non impactées pendant les saisons pluvieuses ; en filigrane Foto et al., (2010) avaient par ailleurs constaté une abondance de taxons au niveau des cours d'eau pendant ces saisons. En effet au niveau de l'effluent, l'indice de similarité de Sørensen (Tableau 4) entre les stations au niveau du périmètre irrigué et la zone intermédiaire de $98,18 \%$ indique que ces stations situées sur l'effluent sont très proches d'un point de vue composition faunistique en macroinvertébrés. Ces stations partagent en plus des 18 familles, 5 autres familles représentées au niveau de la figure 4. Le rapprochement de la station intermédiaire avec la station du fleuve observé au niveau de la figure 2 est sans doute révélateur d'une possible récupération (i.e. une recolonisation de la diversité biologique) de ce cours d'eau. En effet, notre théorie de possible récupération se base d'une part sur les observations de Cissé (1997) qui avait remarqué une diminution de la concentration des produits chimiques au fur et à mesure qu'on s'éloigne des zones d'utilisation anthropiques et d'autre part de la présence dans les échantillons au niveau du fleuve des individus de la famille des Baetidés et des Ephémérellidés (Ordre des Ephéméroptères). L'ordre des Ephéméroptères est présenté par Moisan et Pelletier (2008) comme indicateur de bonne qualité de l'eau.

révèle par ailleurs que la différence entre les diversités de Shannon de ces deux zones s'accentue pendant le mois de juillet, période de pluie au niveau du bassin. Au niveau du continuum lac de barrage-effluent-fleuve, un total de 35 familles a été échantillonné dont 27 au niveau du lac de barrage et des stations proches du périmètre irrigué et 32 au niveau de la station de l'effluent loin du périmètre irrigué et du fleuve. La présence d'individus appartenant à la famille des Baetidés et des Ephémérellidés (ordre des Ephéméroptères) au niveau du fleuve permet de croire à une possible récupération du cours d'eau car cet ordre d'insectes est considéré comme sensible aux perturbations des cours d'eau. 


\section{BIBLIOGRAPHIE}

AFNOR, 2004. L'Indice Biologique Global Normalisé français (IBGN): ses principes et son évolution dans le cadre de la Directive Cadre Européenne sur l'eau. NF T90-350.

AFNOR, 2009. Qualité écologique des milieux aquatiques. Qualité de l'eau. Prélèvement des macroinvertébrés aquatiques en rivières peu profondes. Association française de normalisation, Norme expérimentale T 90-333.

Brown DS, 1980. Freshwater Snails of Africa and their Medical Importance. Taylor and Francis LTD London. 487p.

Cecchi P., Nikiema A., Moiroux N., Sanou B. 2009. Towards an atlas of lakes and reservoirs in Burkina Faso. In Andreini M., Schuetz T., Harrington L. (eds.). Small reservoirs toolkit.IWMI, Battaramulla, Colombo, Sri Lanka, $23 \mathrm{p}$.

Cissé N., 1997. Évaluation hydro-écologique du bassin du Niger Supérieur. Freshwater contamination (Proceedings of Rabat Symposium S4, April-Mai 1997).IAHS Publ. N ${ }^{\circ} 243$. p 27-33.

Davis A.M., Thorburn P.J., Lewis S.E., Bainbridge Z.T., Attard S.J., Milla R. et Brodie J.E., 2011. Environnemental impacts of irrigated sugarcane production: Herbicide run-off dynamics from farms and associated drainage systems. Agric. Ecosyst. Environ. (2011), doi : 10.1016/j.agee.2011.06.019

Déjoux C. 1977. Chironomides du lac de Bam (Haute Volta). Cah. O.R.S.T.O.M., sér. Hydrobiol., vol. XI, no 4, 1977 : 291-295.

Diomande D, Bony YK, Edia EO, Konan KF, Gourene G, 2009. Diversité des macroinvertébrés de la Rivière Agnéby (Côte d'lvoire ; Afrique de I'Ouest). European Journal of Scientific Research 35(3): 368-377.

Foto MS, Zebaze TSH, Nyamsi TN, Njiné T. 2010. Macroinvertébrés Benthiques du cours d'eau Nga: Essai de Caractérisation d'un Référentiel par des Analyses Biologiques. European Journal of ScientificResearch. 43(1) pp.96106

Foto MS, Zebaze TSH, Nyamsi TNL, Ajeagah GA, Njiné T, 2011.Évolution spatiale de la diversité des peuplements de macroinvertébrés benthiques dans un cours d'eau anthropisé en milieu tropical (Cameroun). European Journal of ScientificResearch 55(2): 291-300.
GIRE, 2001 - État des lieux des ressources en eau du Burkina Faso et de leur cadre degestion. Version finale, Mai 2001. Direction Générale des Ressources en Eau. Ouagadougou, Burkina Faso.

Guenda W, 1996. Étude faunistique, écologique et de la distribution des insectes d'un réseau hydrographique de l'Ouest africain : Mouhoun (Burkina Faso); Rapport avec SimuliumdamnasumTheobald, vecteur de l'onchocercose. Thèse Doctorat d'Etat és sciences, Université de Droit, d'Economie et des sciences D'Aix-Marseille III, France, $260 \mathrm{p}$.

Hammer $\varnothing$, Harper DAT, P D Ryan 2001. PAST: Paleontological Statistics Software Package for Education and Data Analysis. PalaeontologiaElectronica 4(1): 9pp.

Kabré TA, Diguingué D, Bouda S, 2002. Effet du rétrécissement de la superficie d'eau sur les macroinvertébrés du lac de barrage de la Comoé, Sud-ouest du Burkina Faso. Science et Technique, série Sciences Naturelles et Agronomie 26(1) : 37-49.

Kouamé MK, Dietoa MY, Edia EO, Da Costa SK, Ouattara A, Gourène G, 2011. Macroinvertebrates communities associated with macrophyte habitats in a tropical man-made lake (Lake Taabo, Cote d'Ivoire). Knowledge and Management of Aquatic Ecosystems. (2011) 400, 03. DOI : 10.105/kmae/2010035

Lafont M. 1983. Introduction pratique à la systématique des organismes des eaux continentales Françaises. 3 : Annélides Oligochètes. Bulletin mensuel de la société Linnéenne de Lyon. 52e année, $n^{\circ} 4:$ : 107-135

Leigh C., Burford M.A., Roberts D.T., Udy J.W., 2010. Predicting the vulnerability of reservoirs to poor water quality and cyanobacterial blooms. Water Research 44: 4487-4496.

Lévêque C et Durand JR 1981. Flore et Faune aquatiques de l'Afrique Sahélo-Soudanienne, Tome II. Editions de l'ORSTOM, Paris, France. $482 \mathrm{p}$.

Marzin A., Archaimbault V., Belliard J., Chauvin C., Delmas F. et Pont D., 2012.Ecological assessment of running waters: Do macrophytes, macroinvertebrates, diatoms and fish show similar responses to human pressures? Ecological indicators (23): pp 56-63. 
Mazzela L., Bortoli J. de, Argillier C., 2009. Création d'un nouvel outil de bioindication basé sur les communautés d'invertébrés benthiques lacustres: méthodes d'échantillonnage et métriques candidates. Rapport d'avancement. Cemagref. Unité de Recherche Hydrobiologique. Équipe Écosystèmes Lacustres. Aix en Provence, France. Mars 2009. 25p.

Merrit RW et Cummins KW, 1984. An introduction to the aquatic insects of north America, second edition, Dubuque (lowa), Kendall//Hunt Publishing Company, USA. 722p.

Moisan J, 2010. Guide d'identification des principaux macroinvertébrés benthiques d'eau douce $\mathrm{du}$ Québec, Surveillance volontaire des cours d'eau peu profonds. Direction du suivi de l'état de l'environnement, ministère du Développement durable, de l'Environnement et des Parcs, Québec, Canada, 82p

Moisan $\mathrm{J}$ et Pelletier L, 2008. Guide de surveillance biologique basée sur les macroinvertébrés benthiques d'eau douce du Québec - Cours d'eau peu profonds à substrat grossier. Direction du suivi de l'état de l'environnement, ministère du Développementdurable, de l'Environnement et des Parcs, Québec, Canada. $86 \mathrm{p}$.

Mouthon J. 1996. Un indice biologique lacustre basé sur l'examen des peuplements de mollusques. Bull. Fr. Pêche Piscic. 66(331). 397-406.

Pequeux A. 2006. La vie dans les abysses océaniques. Bulletin de la Royale des Sciences de Liège. Vol 75. pp 119-141.

Poda JN, Mwanga J, Dianou D, Garba A, Ouédraogo F, Zongo D, Sondo KB, 2006.Les parasitoses qui minent les nouveaux pôles de développement au Burkina Faso : cas desschistosomoses et des géohelminthes dans le complexe hydroagricole du Sourou.VertigO. Volume 27, numéro 2. Septembre 2006.

http : //vertigo.revues.org/2378. Consulté le 02 février 2013.

Sass L.L., Bozek M.A., Hauxwell J.A., Wagner K., Knight S., 2010. Response of aquatic macrophytes to human land use perturbations in the watersheds of Wisconsin lakes, U.S.A. Aquatic Botany 93: 18.

Shannon CE and Weaver V. 1949. The mathematical theory of communication. Urbana, IL: University of Illinois Press.

Sørensen T.A. 1948 .A method of establishing groups of equal amplitude in plant sociology based on similarity of species content, and its application to analyses of the vegetation on Danish commons. Kongelige Danske Videnskabernes Selskabs Biologiske Skrifter, 5, 1-34.

Tachet H, Richoux P, Bournaud M, Usseglio-Polatera P, 2000. Invertébrés d'eau douce. Systématique, Biologie, écologie. CNRS EDITIONS, Paris, France. $588 \mathrm{p}$.

Venot JP et Cecchi P, 2011. Valeurs d'usage ou performances technologiques: comment apprécier le rôle des petits barrages en Afrique subsaharienne? Cahiers Agriculture 20(1-2): 112-117.

Zouggaghe F. et Moali A., 2009. Variabilité structurelle de peuplement de macroinvertébrés benthiques dans le bassin versant de la Soummam (Algérie, Afrique du Nord). Rev. Ecol. (Terre Vie). Vol 64. 305-321.

Wiederholm T. 1980. Use of benthos in lake monitoring. J. Wat. Polut. Control Fed., 52. 537-547. 